FACTA UNIVERSITATIS

Series: Mechanical Engineering Vol. 15, No 2, 2017, pp. 201 - 215

DOI: 10.22190/FUME170203005A

Original scientific paper

\title{
MODELLING AND CONTROL OF H-SHAPED RACING QUADCOPTER WITH TILTING PROPELLERS
}

\author{
UDC 681.5
}

\author{
Ahmed Alkamachi ${ }^{1,2}$, Ergun Ercelebi ${ }^{2}$ \\ ${ }^{1}$ Alkawarizmi College of Engineering, University of Baghdad, Iraq \\ ${ }^{2}$ Department of Electric and Electronic Engineering, Gaziantep University, Turkey
}

\begin{abstract}
Traditional quadcopter suffers terribly from its underactuation which implies the coupling between the rotational and the translational motion. In this paper, we present a quadcopter with dynamic rotor tilting capability in which the four propellers are allowed to tilt together around their arm axis. The proposed model provides leveled forward/backward horizontal motion and therefore, ensures a correct view of the onboard camera, and increases the vehicle speed by reducing the air drag. The rotor tilt mechanism also provides an instant high speed in the forward or reverse direction and offers a quick and solid air brake to restrain that fast moving speed. The nonlinear dynamical model for the quadcopter under consideration is derived using NewtonEuler formalization. A control strategy is then proposed aimed to control the altitude, attitude, and the forward speed of the obtained model. Finally, a numerical simulation is used to integrate the system model with the controller and to test the system performance. Simulation results are reported to demonstrate the advantages of the proposed novel configuration.
\end{abstract}

Key Words: Genetic Algorithm, Newton-Euler Formalization, PID Controller, Racing Quadcopter, Tilt Rotor

\section{INTRODUCTION}

In the recent years, unmanned aerial vehicles (UAVs) have gained a considerable attention for their applications in scientific, civilian, and military fields. Quadcopter UAV is defined as a small vehicle with four rotor-propeller sets distributed around its body [1]. It is a highly nonlinear, multi input multi output (MIMO), extremely coupled and underactuated system [2]. The quadcopter has become one of the most popular UAV designs due to its vertical take-off

Received February 3, 2017 / Accepted April 27, 2017

Corresponding author: Ahmed Alkamachi

Affiliation: Alkawarizmi College of Engineering, University of Baghdad, Katir Alnada St., AlJadiriyah, Baghdad, Iraq

E-mail: amrk1978@gmail.com

() 2017 by University of Niš, Serbia | Creative Commons Licence: CC BY-NC-ND 
and landing (VTOL) property, simplicity in mechanical configuration, and ease of maintenance. Furthermore, the use of four smaller propellers in the quadcopters reduces the danger posed by the propellers if they touch an external object as compared with one big propeller in helicopter or airplane [3]. The racing quadcopters with H-shape configuration have been gaining an increasing attention due to their use in several critical applications like surveillance, search, rescue, firefighting, and UAV-based delivery.

Classical quadcopters can control their position and orientation by altering the rotors' spinning speeds. For example, if the quadcopter is required to roll, it would make a difference between the right and left propellers' speeds. In a similar manner, the desired pitch angle can be achieved by changing the relative speeds of the front and rear rotors [4]. For the translational motion, the quadcopter needs to roll for lateral motion and to pitch for forward/backward motion. This coupling between the quadcopter states has the undesired influence of changing the onboard surveillance camera viewing axis, so it limits the quadcopter ability to do some vision-based tasks [5].

Considerable academic references have been reviewed to understand the current state of the art in the quadcopter design. For the purpose of coping with the underactuation problem, several prospects have been suggested through the reviewed literature spanning different techniques in thrust vectoring concepts, and new mechanical configurations. Many gaps have been filled and several new novel designs have been proposed aiming to improve the traditional quadrotor performance.

In [6], the authors propose a quadcopter with four tiltable wings distributed under its rotors. The modified quadcopter can change its mode from quadcopter to aircraft and vice versa by tilting its wings all at once. M. K. Mohamed et al. propose a novel tri-tilt-rotor UAV in which the three rotors can be tilted independently to gain a full authority and control over the vehicle states [7]. Following a similar concept, the rotor tilt mechanism has been utilized in several studies to resolve the coupling problem between the quadcopter position and orientation. For instance, M. Ryll et al. design and construct a quad tilt rotors mini quadcopter that converts the traditional quadcopter into an overactuated air vehicle [8]. Another solution to resolve the fundamental underactuation limitations of the quadrotor is made by adding one degree of freedom to each of the quadcopter rotors [9]. Looking for further improvement, Fernandes presents a quadcopter with 2-axis tilting mechanism added to two of its rotors [10]. For the purpose of more maneuverability and robustness, the authors in $[5,11,12]$ propose a quadcopter with a novel 2 -axis tilting mechanism in which the rotors have two degrees of freedom. Badr et al. introduce a modified quadcopter that allows each rotor to tilt about the axes perpendicular to its arm [13].

In this paper, an $\mathrm{H}$-shaped racing quadcopter with tiltable rotors is introduced. To the authors' best knowledge this configuration is novel and its dynamical model has not been obtained yet. The four rotors; which are ordinarily fixed, are allowed to rotate around their arm axes simultaneously. With this tilting capability, the number of control inputs is increased to five (the four rotors spinning speeds plus the tilt angle). The additional control input is used to govern the forward/ backward speed of the proposed model.

The main contributions of this work are: (1) To derive a complete dynamical model for the H-shaped racing quadcopter with tilting propellers, (2) To propose and design a tracking control aimed at exploring the rotor tilt advantages, (3) To conceptualize the tilt mechanism and thrust vectoring concept. 
The paper is outlined as follows: The mathematical model is derived in Section 2. The controller is designed and tuned in Section 3. A comprehensive set of numerical simulation tests is then carried out in Section 4. Finally, the concluding remarks are given in Section 5.

\section{SYSTEM MODELING}

The aim of this section is to develop a dynamical model for the H-shaped tilt rotors racing quadcopter so as to define the ratio that relates the quadcopter states with the propellers spinning speed and the rotor tilt angle. At this stage, it is important to state some acceptable hypotheses that are used to simplify the process of obtaining the system dynamics. Without these hypotheses, the system mathematical model will be complicated and difficult to obtain [14]. These hypotheses are:

1. The system is assumed to be symmetric and rigid.

2. The quadrotor body frame origin and the centre of gravity $(\mathrm{CoG})$ are coinciding.

3. The actuators are assumed to have sufficient fast response, so their dynamics are neglected [15].

\subsection{Model configuration}

The tilt rotor H-shaped racing quadcopter will be shortened as (Hcopter) throughout the following sections. The proposed model consists of an H-shaped frame with four rotors distributed at the frame tips as shown in Fig. 1a. The rotors are allowed to tilt simultaneously around the arms connecting them to the main frame in the range of $-\pi / 2<\alpha<\pi / 2$. Fig. $1 \mathrm{~b}$ shows the rotor tilt angle.

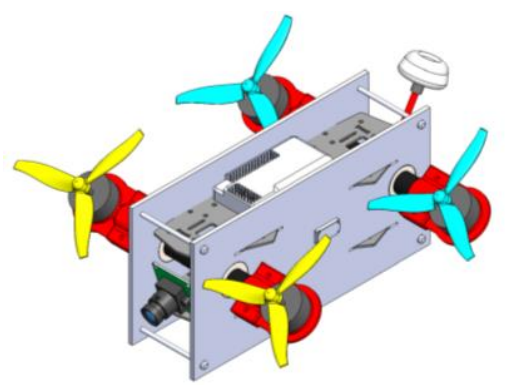

a)

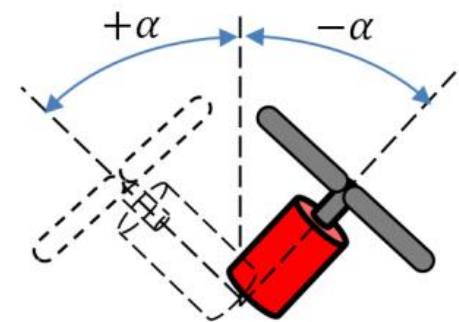

b)

Fig. 1 Hcopter configuration: a) Model CAD drawing; b) Tilt rotor angle

\subsection{Coordinate Systems}

First, let $F^{E}:\left\{X^{E}, Y^{E}, Z^{E}\right\}$ being the earth frame and $F^{B}:\left\{X^{B}, Y^{B}, Z^{B}\right\}$ being the base frame (see Fig. 2) with its center coinciding with the vehicle's CoG. It is necessary to define these frames since some quantities should be expressed in $F^{B}$ (for instance the rotors' generated thrusts) while the other should be defined in $F^{E}$ (the gravitational force). A superscript letter " $B$ " is assigned to the variables that belong to $F^{B}$, while " $E$ " lettered superscript is used to denote the variables resolved in $F^{E}$. 


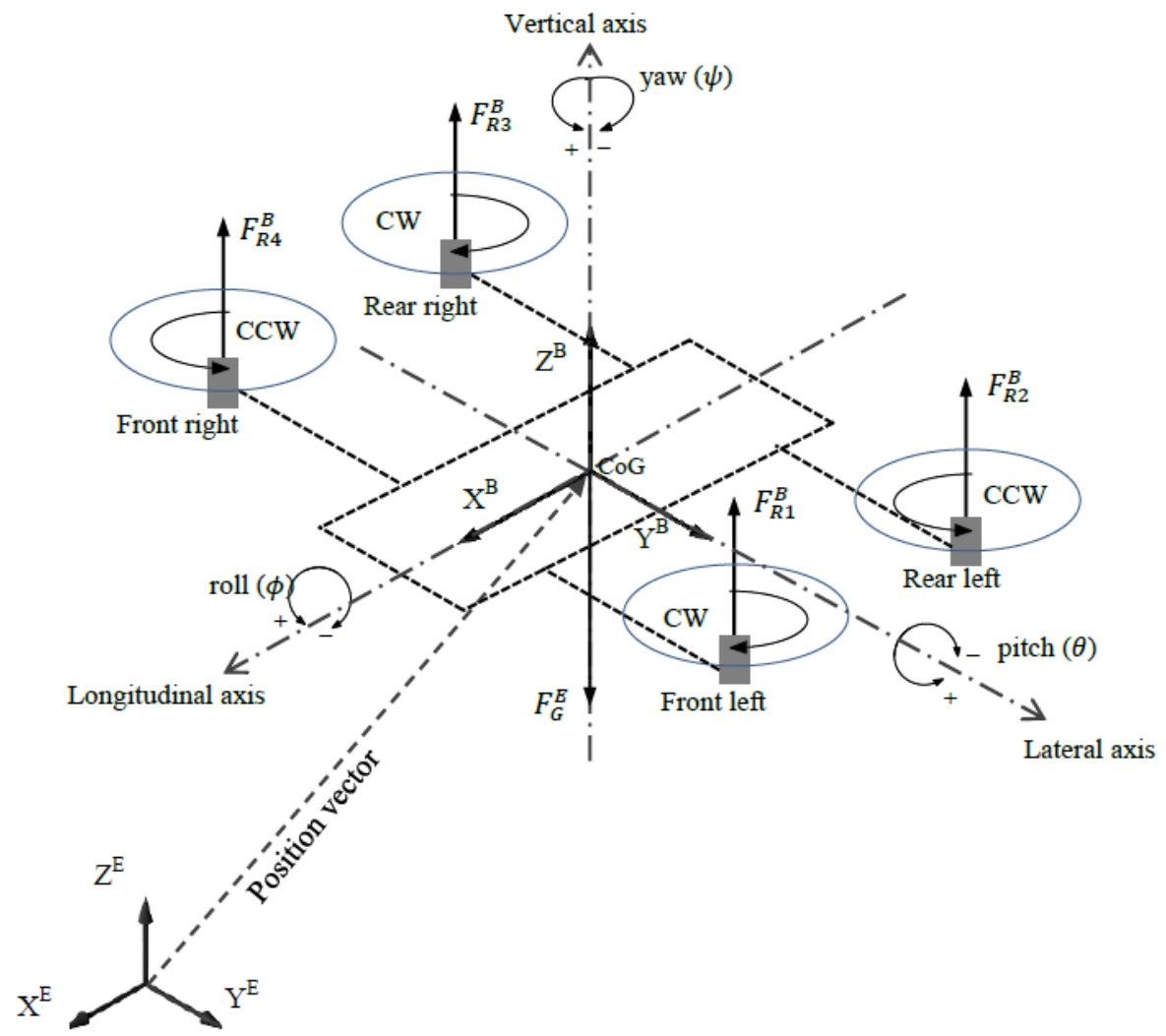

Fig. 2 The system model schematic showing the reference frames, Euler angles, and axes

To go from $F^{E}$ to $F^{B}$, a rotation matrix should be introduced. The rotation matrix (also called the direction cosine matrix), is a result of multiplying three canonical rotation matrices $R_{X}(\varphi), R_{Y}(\theta)$, and $R_{Z}(\psi)$ with a specific sequence [16], where $\varphi$ (roll), $\theta$ (pitch), and $\psi$ (yaw) are the NASA based Euler angles [17]. It follows that:

$$
\begin{gathered}
{ }^{B} R_{E}=R_{X}(\varphi) * R_{Y}(\theta) * R_{Z}(\psi) \\
=\left[\begin{array}{ccc}
C_{\theta} C_{\psi} & C_{\theta} S_{\psi} & -S_{\theta} \\
-C_{\varphi} S_{\psi}+S_{\varphi} S_{\theta} C_{\psi} & C_{\phi} C_{\psi}+S_{\varphi} S_{\theta} S_{\psi} & S_{\varphi} C_{\theta} \\
S_{\varphi} S_{\psi}+C_{\varphi} S_{\theta} C_{\psi} & -S_{\varphi} C_{\psi}+C_{\varphi} S_{\theta} C_{\psi} & C_{\varphi} C_{\theta}
\end{array}\right]
\end{gathered}
$$

where ${ }^{B} R_{E}$ is the orientation matrix of the variables in $F^{E}$ with respect to $F^{B}$. The $C$ and $S$ are the sine and cosine functions, respectively.

For the reverse operation (transferring the variables from $F^{B}$ to $F^{E}$ ), an inverse matrix ${ }^{E} R_{B}=\left({ }^{B} R_{E}\right)^{-1}$ should be used. Since the orientation matrix is a result of orthogonal matrices multiplication, then its inverse is just its transpose [16].

$$
{ }^{E} R_{B}=\left({ }^{B} R_{E}\right)^{-1}=\left({ }^{B} R_{E}\right)^{T}
$$




\subsection{Static and Dynamic Model}

In this section, all the dominant forces and torques that act on the Hcopter body are discovered.

\subsubsection{Forces}

Rotors' generated forces ${ }^{B} F_{T R}$ : Assume that the $i^{\text {th }}$ propeller spinning speed is denoted by $\Omega_{\mathrm{i}}$. Then, according to the blade element theory $[18,19]$, the generated force in the $\mathrm{z}$-direction is given by $k\left(\Omega_{\mathrm{i}}\right)^{2}$, where $k$ is the rotor thrust coefficient. When the rotor tilts with an angle $\alpha$, then the generated force can be resolved into its components along the $\mathrm{x}$ and $\mathrm{z}$ axes. It follows that the $i^{\text {th }}$ rotor generated force is:

$$
{ }^{B} F_{R i}=\left[\begin{array}{c}
k \Omega_{i}^{2} \sin (\alpha) \\
0 \\
k \Omega_{i}^{2} \cos (\alpha)
\end{array}\right]
$$

and the total generated force due to the four spinning propellers is:

$$
{ }^{B} F_{T R}=\sum_{i=1}^{4}{ }^{B} F_{R i}=K U
$$

where

$$
\mathrm{K}=\left[\begin{array}{llllllll}
k & 0 & k & 0 & k & 0 & k & 0 \\
0 & 0 & 0 & 0 & 0 & 0 & 0 & 0 \\
0 & k & 0 & k & 0 & k & 0 & k
\end{array}\right]
$$

is the thrust coefficient matrix, and

$$
U=\left[\Omega_{1}^{2} * S_{\alpha}, \Omega_{1}^{2} * C_{\alpha}, \Omega_{2}^{2} * S_{\alpha}, \Omega_{2}^{2} * C_{\alpha}, \Omega_{3}^{2} * S_{\alpha}, \Omega_{3}^{2} * C_{\alpha}, \Omega_{4}^{2} * S_{\alpha}, \Omega_{4}^{2} * C_{\alpha}\right]^{T}
$$

is the control input vector, $\mathrm{S}_{\alpha}$ and $\mathrm{C}_{\alpha}$ are the $\sin (\alpha)$ and $\cos (\alpha)$ function respectively.

Gravitational force ${ }^{E} F_{G}$ : According to the Newton's law of universal gravitation, this force tries to pull down the vehicle toward the earth and it can be expressed in $F^{E}$ by:

$$
{ }^{E} F_{G}=\left[\begin{array}{c}
0 \\
0 \\
-m g
\end{array}\right]
$$

where $\mathrm{g}$ is the gravitational constant, and $m$ is the vehicle total mass.

Drag reluctant force ${ }^{B} F_{D}$ : It is a result of the air friction with the quadcopter body in motion and it is directly proportional to the vehicle moving speed.

$$
{ }^{B} F_{D}=-K_{d} \dot{P}^{B}
$$

where $K_{d}$ is the $3 \times 3$ aerodynamic coefficient matrix, and $\dot{P}^{B}=[\dot{x}, \dot{y}, \dot{z}]^{T}$ is the Hcopter velocity vector which represents the time derivative of quadcopter body position vector $P^{B}$. 
Total force ${ }^{B} F_{T}$ : The total force acting on the quadcopter body is the vector sum of the above three individual forces.

$$
{ }^{B} F_{T}={ }^{B} F_{T R}+{ }^{B} R_{E} *{ }^{E} F_{G}+{ }^{B} F_{D}
$$

\subsubsection{Torques}

Rotors' generated torque ${ }^{B} M_{T R}$ : It is a result of the four rotor's generated force around the CoG. At this point, it is assumed that the origin of $F^{B}$ and quadcopter $\mathrm{CoG}$ coincide. The total moment due to the rotors' generated forces is:

$$
{ }^{B} M_{T R}=\sum_{i=1}^{4}\left(L_{i} \times{ }^{B} F_{R i}\right)
$$

where $L_{i}$ being a vector directed from the $\mathrm{CoG}$ to the $i^{\text {th }}$ rotor, i.e.:

$L_{l}=\left[L_{x}, L_{y}, 0\right]^{T}, L_{2}=\left[-L_{x}, L_{y}, 0\right]^{T}, L_{3}=\left[-L_{x},-L_{y}, 0\right]^{T}$, and $L_{4}=\left[L_{x},-L_{y}, 0\right]^{T}$, and $L_{x}, L_{y}$ are shown in Fig. 3.

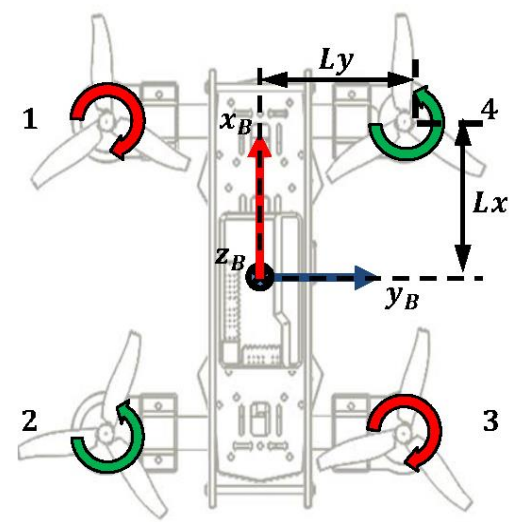

Fig. 3 Hcopter schematic

Aerodynamic drag torque ${ }^{B} M_{D T}$ : It is the counter rotating torque due to the air drag caused by propeller spinning [20]. According to the blade element theory $[18,19]$, the $i^{\text {th }}$ rotor's drag torque around the $\mathrm{z}$-axis can be expressed as $(-1)^{i} b\left(\Omega_{\mathrm{i}}\right)^{2}$, where $b$ is the rotor drag coefficient and the factor $(-1)^{i}$ is negative for the propellers rotating clockwise (CW) (rotors 1 and 3 ) and positive for those rotating in a counter clockwise (CCW) direction (rotors 2 and 4). Recall that the rotors can be tilted with angle $\alpha$, then the drag torque of the $i^{\text {th }}$ propeller is resolved into $\mathrm{x}$ and $\mathrm{z}$ components as follows:

$$
{ }^{B} M_{D i}=\left[\begin{array}{c}
(-1)^{i} b \Omega_{i}^{2} \sin (\alpha) \\
0 \\
(-1)^{i} b \Omega_{i}^{2} \cos (\alpha)
\end{array}\right]
$$

so the total drag torque for the four propellers is:

$$
{ }^{B} M_{D T}=\sum_{i=1}^{4}{ }^{B} M_{D i}
$$


Total torque ${ }^{B} M_{T}$ : Expressed in $\mathrm{F}^{\mathrm{B}}$, the total torque acting on the Hcopter body is the vector sum of the above two individual torques.

where

$$
{ }^{B} M_{T}={ }^{B} M_{T R}+{ }^{B} M_{D T}=B U
$$

$$
\mathrm{B}=\left[\begin{array}{cccccccc}
-b & k L_{y} & b & k L_{y} & -b & -k L_{y} & b & -k L_{y} \\
0 & -k L_{x} & 0 & k L_{x} & 0 & k L_{x} & 0 & -k L_{x} \\
-k L_{y} & -b & -k L_{y} & b & k L_{y} & -b & k L_{y} & b
\end{array}\right]
$$

is the moment coefficient matrix, and $U$ is the control input vector as before.

\subsubsection{Virtual control vector $\boldsymbol{V}$}

At this phase of the mathematical modelling and for the purpose of better understanding, it is important to define a virtual control vector $\boldsymbol{V}=\left[V_{1}, V_{2}, V_{3}, V_{4}\right]^{\mathrm{T}}$. First virtual control input $V_{l}$ is in charge of controlling the vehicle altitude since it represents the resultant lifting forces generated by the rotors in the upward positive $\mathrm{z}$ direction.

$$
V_{1}={ }^{B} F_{T R z}=k\left(\Omega_{1}^{2}+\Omega_{2}^{2}+\Omega_{3}^{2}+\Omega_{4}^{2}\right) \cos (\alpha)
$$

Second virtual control input $V_{2}$ is the total torque around $\mathrm{x}$-axis; thus it is responsible for controlling the roll angle.

$$
V_{2}={ }^{B} M_{T x}=b\left(-\Omega_{1}^{2}+\Omega_{2}^{2}-\Omega_{3}^{2}+\Omega_{4}^{2}\right) \sin (\alpha)+k L_{y}\left(\Omega_{1}^{2}+\Omega_{2}^{2}-\Omega_{3}^{2}-\Omega_{4}^{2}\right) \cos (\alpha)
$$

Third virtual control input $V_{3}$ represents the total torque around y-axis so it controls the pitch angle of the Hcopter.

$$
V_{3}={ }^{B} M_{T y}=k L_{x}\left(-\Omega_{1}^{2}+\Omega_{2}^{2}+\Omega_{3}^{2}-\Omega_{4}^{2}\right) \cos (\alpha)
$$

Fourth virtual control input $V_{4}$ is responsible for adjusting the yaw angle since it represents the total torque around $\mathrm{z}$-axis.

$$
V_{4}={ }^{B} M_{T z}=k L_{y}\left(-\Omega_{1}^{2}-\Omega_{2}^{2}+\Omega_{3}^{2}+\Omega_{4}^{2}\right) \sin (\alpha)+b\left(-\Omega_{1}^{2}+\Omega_{2}^{2}-\Omega_{3}^{2}+\Omega_{4}^{2}\right) \cos (\alpha)
$$

In addition to the above virtual control signals, rotor tilt angle $\alpha$ is used to control the forward/backward speed of the vehicle. When the rotors tilt, they provide the required horizontal force to move the Hcopter along the $\mathrm{x}$-axis direction.

Equations (11) through (14) can be combined into one matrix equation. It follows that:

$$
V=\Gamma U
$$

where $\Gamma$ is the coefficient matrix and is equal to:

$$
\Gamma=\left[\begin{array}{cccccccc}
0 & k & 0 & k & 0 & k & 0 & k \\
-b & k L_{y} & b & k L_{y} & -b & -k L_{y} & b & -k L_{y} \\
0 & -k L_{x} & 0 & k L_{x} & 0 & k L_{x} & 0 & -k L_{x} \\
-k L_{y} & -b & -k L_{y} & b & k L_{y} & -b & k L_{y} & b
\end{array}\right]
$$




\subsubsection{Model dynamics}

With a view to obtain the quadcopter dynamic and the equations of motion, we exploit the typical Newton-Euler formalization. Recall that $P^{B}$ represents the Hcopter position vector expressed in $F^{B}$, then the Newton-Euler equations are:

$$
m \ddot{P}^{B}={ }^{B} F_{T}
$$

and

$$
J \dot{\omega}^{B}+\left(\omega^{B} \times J \omega^{B}\right)={ }^{B} M_{T}
$$

where $J \in R^{3 \times 3}$ is the moment of inertia tensor, $\omega^{B}$ is the body angular velocity vector, and $\dot{\omega}^{B}$ is the angular acceleration of the quadcopter body expressed in $F^{B}$.

Substituting Eq. (7) in Eq. (17), we can get the linear acceleration vector expressed in $F^{B}$ as:

$$
\ddot{P}^{B}=\frac{1}{m}\left(K U+{ }^{B} R_{E}{ }^{E} F_{G}-K_{d} \dot{P}^{B}\right)=\frac{1}{m}\left(\left[\begin{array}{c}
{ }^{B} F_{T R x} \\
0 \\
V_{1}
\end{array}\right]+{ }^{B} R_{E}{ }^{E} F_{G}-K_{d} \dot{P}^{B}\right)
$$

Angular acceleration $\dot{\omega}^{B}$ can be obtained by substituting Eq. (11) in Eq. (18):

$$
\dot{\omega}^{B}=\ddot{O}^{B}=J^{-1}\left(B U-\left(\omega^{B} \times J \omega^{B}\right)\right)=J^{-1}\left(\left[\begin{array}{l}
V_{2} \\
V_{3} \\
V_{4}
\end{array}\right]-\left(\omega^{B} \times J \omega^{B}\right)\right)
$$

where $O^{B}$ represents the vehicle orientation vector expressed in the body coordinate $\left(O^{B}=[\phi, \theta, \psi]^{T}\right)$, therefore $\left(\ddot{O}^{B}=[\ddot{\varphi}, \ddot{\theta}, \ddot{\psi}]^{T}\right)$.

To this end, we have obtained the Hcopter dynamical equations that govern its operation.

\section{CONTROLler DESIGN}

In this work, the (Matlab/Simulink) environment is used to integrate and examine the obtained model. It is also used to tune the PID parameters using the genetic algorithm (GA) and to carry out all the subsequent tests. The simulation environment is set up on a personal computer with $2.5 \mathrm{GHz}$ processing speed and 6 GB RAM, running on Windows 10. The complete control system block diagram for the Hcopter is shown in Fig. 4. The list of model physical parameters used through all the tests is given in Table 1.

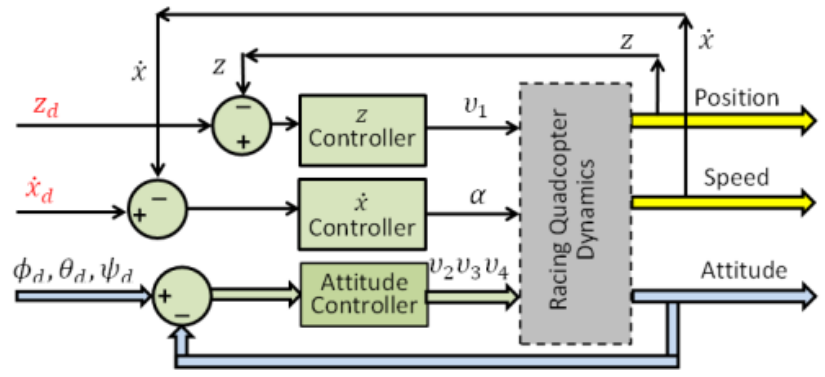

Fig. 4 The proposed controller block diagram 
Table 1 The proposed Hcopter model physical parameters

\begin{tabular}{ll}
\hline Parameters & Value- \\
\hline$m$ & $1.2 \mathrm{Kg}$. \\
$L_{X}, L_{Y}$ & $20 \mathrm{~cm}$ \\
$k, b$ & $3 \mathrm{e}-6 \mathrm{~N} \cdot \mathrm{sec}^{2} / \mathrm{rad}^{2}, 1 \mathrm{e}-7 \mathrm{Nm} \cdot \mathrm{sec}^{2} / \mathrm{rad}^{2}$ \\
$J$ & $\operatorname{diag}[0.02,0.02,0.04]^{*} \mathrm{Kg} \cdot \mathrm{m}^{2}$ \\
$K_{d}$ & $\operatorname{diag}[0.3,0.3,0.5]^{*}$ \\
$\Omega_{\min }, \Omega_{\max }{ }^{* *}$ & $100,2000 \mathrm{rad} / \mathrm{sec}$ \\
${ }^{* *} \operatorname{diag}[$ i is a diagonal matrix \\
${ }^{*}$ It is the rotor's upper and lower speed limit respectively
\end{tabular}

For efficient surveillance tasks, it is important that the quadcopter has a precise control on its attitude, speed, and altitude [11]. The control problem addressed in this work is an output tracking problem. A five output PID control system is proposed to control the orientation (roll, pitch, and yaw), altitude, and the forward speed of this novel air vehicle.

\subsection{Forward/backward speed control}

The additional control input (tilt angle $\alpha$ ) is used to control Hcopter forward speed which in turns improves the surveillance based tasks. The speed error signal is obtained by subtracting desired $x$ speed $\left(\dot{x}_{d}\right)$ from real Hcopter speed $(\dot{x})$. The error signal is then fed to a PID controller that determines the required tilt angle $(\alpha)$ to achieve the desired speed.

\subsection{Altitude control}

The altitude error signal is formed by subtracting the measured altitude (z position) from desired elevation $\mathrm{Z}_{d}$. It is then applied to the PID controller that adjusts the value of control input $V_{1}$ to achieve the required altitude.

\subsection{Orientation control}

The orientation controller is the core of the control system and it is of critical importance. It consists of three PID controllers to keep the quadcopter attitude to the required roll $\left(\varphi_{d}\right)$, pitch $\left(\theta_{d}\right)$ and yaw $\left(\psi_{d}\right)$ angles by controlling the three virtual control signals $V_{2}, V_{3}$, and $V_{4}$, respectively.

\subsection{Virtual control to rotors' speeds}

In this block, the determined rotor tilt angle $(\alpha)$ and the virtual control vector $(V)$ are used to determine the proper rotors' spinning speeds with the aid of Eq. (16).

\subsection{PID parameters tuning using GA}

Genetic algorithm is a search heuristic process that simulates the natural selection procedure [21]. The algorithm is used to tune the five PID parameters by minimizing the following cost function:

$$
\text { Obj } \left.=\left.e r r\right|_{\left(\Omega_{\min }<\Omega_{i}<\Omega_{\text {max }}\right.} \text { for } i=1,2,3,4\right)
$$

where err is the difference between the desired and the actual output response. 
The tuning process is subjected to actuator upper and lower constraints as noted in Eq. (21). It means that if the selected PID parameters lead to excessive control signal output that cause the actuators to exceed their upper and lower limits, then the cost function value for these selected parameters is assigned to an extremely large weight so that it will be excluded from the next iteration. The genetic algorithm takes the response data from the Simulink model to evaluate the cost function and to select the optimal PID parameters. The obtained PID parameters with their associated step response characteristics (settling time $t_{s}$ and percentage peak overshoot $M_{P}$ ) are tabulated in Table 2.

Table 2 PID parameters and step response characteristics

\begin{tabular}{lccccl}
\hline $\begin{array}{l}\text { Controlled state } \\
\text { (Initial } \longrightarrow \text { Final })\end{array}$ & $K_{P}$ & $K_{I}$ & $K_{D}$ & $\begin{array}{l}t_{s} \\
\text { Sec. }\end{array}$ & $\begin{array}{l}M_{P} \\
\%\end{array}$ \\
\hline$z_{d}(0 \longrightarrow 1 m)$. & 22.2 & 22.4 & 7.1 & 1.18 & 1.5 \\
$\varphi_{d}(0 \longrightarrow 5$ deg. $)$ & 17.5 & 1.04 & 0.78 & 0.16 & 0.3 \\
$\theta_{d}(0 \longrightarrow$ 5deg. $)$ & 17.5 & 1.04 & 0.78 & 0.16 & 0.3 \\
$\psi_{d}(0 \longrightarrow 10$ deg. $)$ & 1.63 & 0.04 & 0.25 & 0.43 & 0.4 \\
$\dot{x_{d}}(0 \longrightarrow 2 m /$ sec. $)$ & 0.99 & 0.99 & 0.05 & 2.11 & 7.8 \\
\hline
\end{tabular}

\section{SiMULATION RESULTS}

In order to check the validity of the proposed model and controller, a Simulink model is developed. The purpose of the simulation is to highlight the Hcopter capabilities and the dynamic rotor tilting advantages. The simulation process falls into two phases. The first simulation assumes the ideal case and the second simulation assumes the existence of sensor noise, parameters uncertainties, and external disturbances.

\subsection{Ideal case}

Altitude and attitude tracking: In this test, it is assumed that the Hcopter is initially at rest $\left(P^{E}=[0,0,0]^{\mathrm{T}}, O^{B}=[0,0,0]^{\mathrm{T}}\right)$ and it is then ordered to climb to 1 meter $(\mathrm{z}=1 \mathrm{~m}$. $)$. The vehicle is then commanded to follow the following desired attitude $\left(\varphi_{d}=5 \mathrm{deg}\right.$. at $t=1 \mathrm{sec}$., $\theta_{d}=$ $-5 \mathrm{deg}$. at $t=2 \mathrm{sec}$.,$\psi_{d}=10 \mathrm{deg}$. at $t=3 \mathrm{sec}$.). The simulation results for the desired step inputs are shown in Fig. 5, while the step input characteristics for this test are given in Table 2.

Hcopter speed control: In this part of the simulation, the Hcopter is assumed to be hovering at $\mathrm{z}=1$ meter, and it is required that the body roll, pitch and yaw angles should be kept at zero degree.

Test 1: The Hcopter in this test is examined for its ability to maintain a constant speed while keeping its body leveled $\varphi_{d}=\theta_{d}=0 \mathrm{deg}$. The speed throttle is applied gradually simulating a ramp input from rest to $10 \mathrm{~m} / \mathrm{sec}(36 \mathrm{Km} / \mathrm{h})$ in 5 seconds as shown in Fig. 6a. The simulation results in Figs. $6 \mathrm{~b}$ and $6 \mathrm{c}$ show that the Hcopter can track the desired speed efficiently while maintaining zero attitudes which is impossible for conventional quadcopter to do [22].

Test 2: The aim of this test is to find the maximum forward moving speed that our proposed Hcopter can reach. The tilt angle is increased gradually from $0 \mathrm{deg}$ to its maximum 
allowable limit (45 deg.) in $10 \mathrm{sec}$. It can be seen from Fig. 7 that the maximum reachable speed is approximately $39 \mathrm{~m} / \mathrm{sec}(140 \mathrm{Km} / \mathrm{h})$.

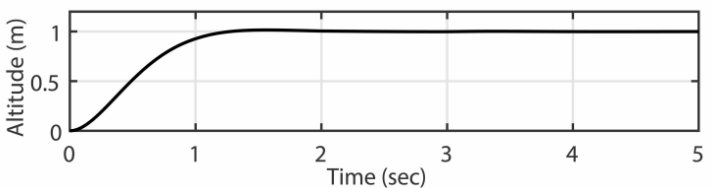

a)

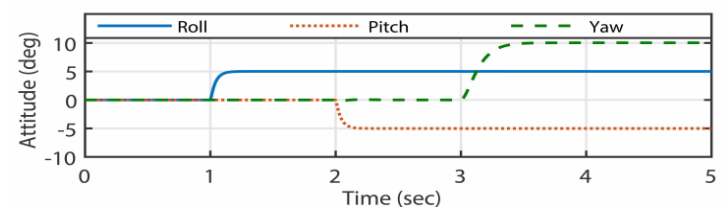

b)

Fig. 5 Step input test simulation results: a) Altitude response; b) Attitude response

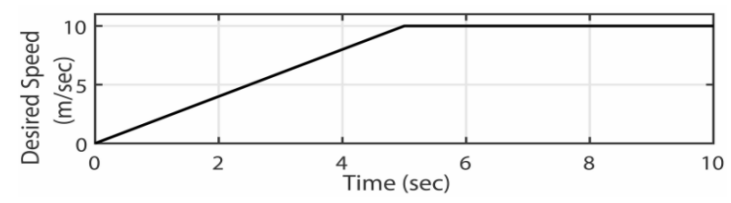

a)

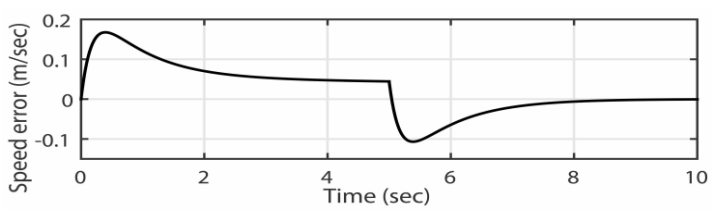

b)

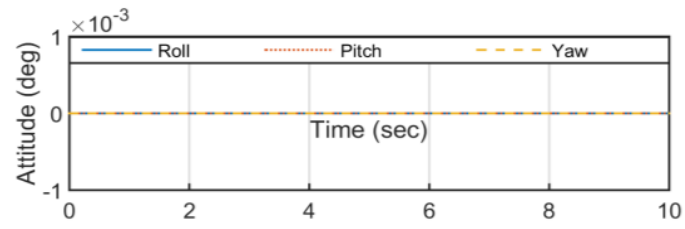

c)

Fig. 6 Speed control test simulation results:

a) Desired speed; b) Speed error; c) Attitude behavior

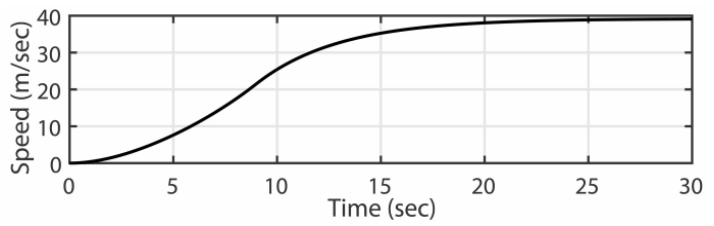

Fig. 7 Hcopter maximum speed test simulation result 
Air braking: From the previous test, it can be noticed that the proposed quadcopter could reach a very high forward speed in a relatively short period; thus, a solid brake is required to rein the vehicle efficiently at the proper time. Fortunately, the rotor tilt mechanism offers an instant braking system that reduces the vehicle speed to zero in a very short period. In this test, the Hcopter forward speed is increased to $10 \mathrm{~m} / \mathrm{s}$ and then suddenly reduced to zero. From Fig. 8a, it can be seen that the rotors tilted with a positive angle during the acceleration period and instantaneously flipped to the opposite side to provide the necessary horizontal opposite force to stop the vehicle. The Hcopter speed behavior is shown in Fig. 8b.

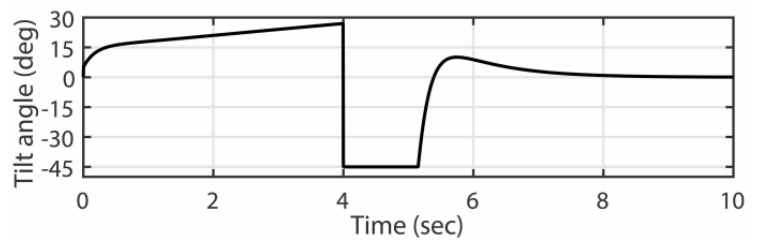

a)

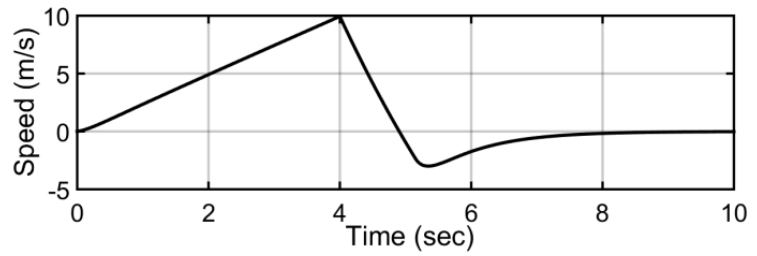

b)

Fig. 8 Air braking test simulation results: a) Tilt angle behavior; b) Vehicle speed

\subsection{Non-ideal case}

In the non-ideal case, three tests were made to examine the model validity and the controller robustness with the existence of white Gaussian sensor noise, external disturbances, and model parameters uncertainty.

Sensor noise: The Hcopter is assumed to be hovering horizontally at $z=1$ meter. A white Gaussian noise with zero mean (shown in Fig. 9a) is applied to the model in the feedback loop to simulate the sensor noise.

The simulation results in Figs. $9 \mathrm{~b}$ and $9 \mathrm{c}$ reveal the controller ability of suppressing the sensor noise successfully. The maximum error in the altitude is just a few millimeters and the maximum drift in the orientation is bounded by $+/-0.01$ degree.

External disturbance: The Hcopter is assumed to be hovering horizontally at $\mathrm{z}=1$ meter and it is commanded to travel with $10 \mathrm{~m} / \mathrm{sec}(36 \mathrm{Km} / \mathrm{h})$ in the forward direction with the existence of opposite light wind with a fixed speed of $1.2 \mathrm{~m} / \mathrm{sec}(4.5 \mathrm{Km} / \mathrm{h})$ [23].

The simulation result shown in Fig. 10 asserts the controller effectiveness in coping with the external disturbances. 


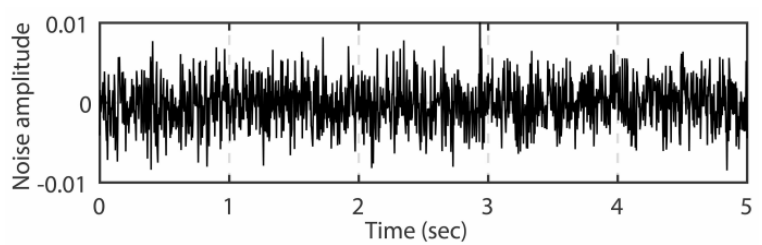

a)

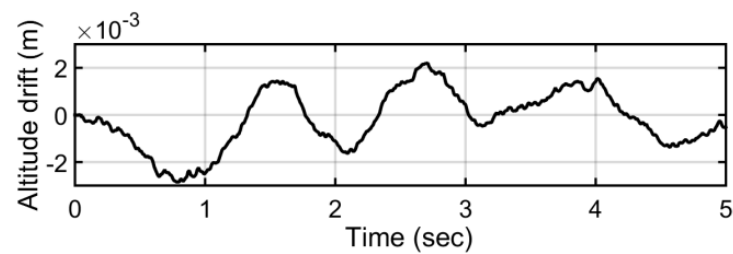

b)

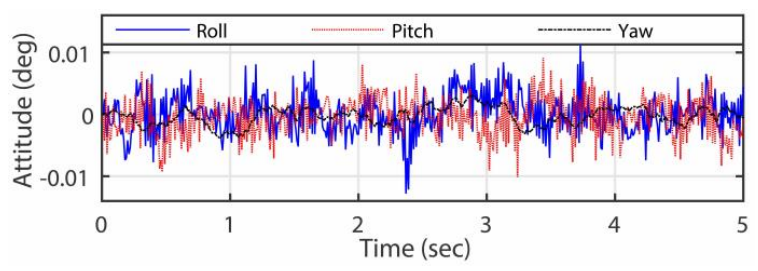

c)

Fig. 9 Noise suppression test simulation results:

a) Noise signal; b) Altitude drift; c) Attitude drift

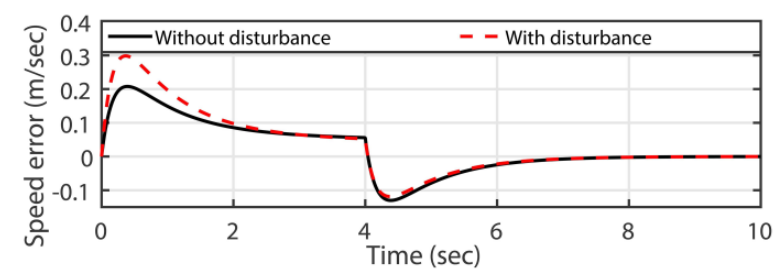

Fig. 10 Hcopter speed error with and without the effect of a light wind disturbance

Model parameters uncertainties: To check the controller effectiveness in dealing with the model uncertainties, the vehicle is ordered to change its altitude from 1 to 2 meter with and without the existence of a $0.5 \mathrm{Kg}$. payload. The payload is suspended from the $\mathrm{CoG}$ of the model. A comparison between the results (see Fig. 11) for both cases shows that the controller performed well against the external additive weight.

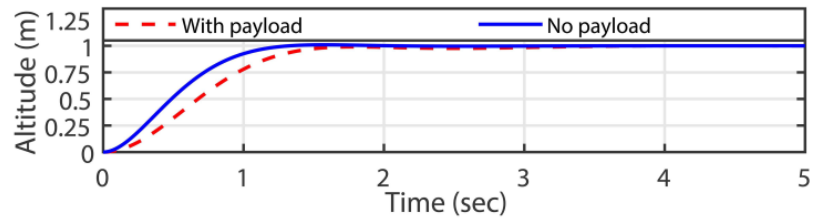

Fig. 11 Uncertainty test simulation results 


\section{CONCLUSION}

In this paper, we have addressed the modeling and control of a novel tilt rotor $\mathrm{H}$-shaped racing quadcopter. Compared to the traditional quadcopter, the proposed model offers higher moving speed with instant air brake. The new design resolves an important part of the underactuation problem in the traditional quadcopter where the translational and rotational motion cannot be controlled independently. The Hcopter, in contrast, allows controlling the rotors thrust direction, therefore granting additional control input results in the uncoupling between the pitch and the forward motion. Several simulation tests have been carried out and the results have been demonstrated and discussed to evaluate the proposed controller validation. By obtaining these encouraging results, the next stage is to design, build, and control the Hcopter prototype.

\section{REFERENCES}

1. Salih, A.L., Moghavvemi, M., Mohamed, H.A.F., Gaeid, K.S., 2010, Modelling and pid controller design for a quadrotor unmanned air vehicle, IEEE Int. Conf. on Automation Quality and Testing Robotics (AQTR), 1, pp. $1-5$.

2. Mian, A.A., Wang, D.B., 2008, Dynamic modeling and nonlinear control strategy for an underactuated quad rotor rotorcraft, Journal of Zhejiang University-Science A, 9(4), pp. 539-545.

3. Nemati, A., Kumar, M., 2014, 2014, Modeling and control of a single axis tilting quadcopter, IEEE American Control Conference (ACC), pp. 3077-3082.

4. Bouabdallah, S., Noth, A., Siegwart, R., 2004, PID vs LQ control techniques applied to an indoor micro quadrotor, IEEE/RSJ International Conference on Intelligent Robots and Systems (IROS 2004), 3, pp. 24512456.

5. Şenkul, F., Altuğ, E., 2013, Modeling and control of a novel tilt —Roll rotor quadrotor UAV, IEEE International Conference on Unmanned Aircraft Systems (ICUAS), pp. 1071-1076.

6. Çetinsoy, E., Dikyar, S., Hançer, C., Oner, K.T., Sirimoglu, E., Unel, M., Aksit, M.F., 2012, Design and construction of a novel quad tilt-wing UAV, Mechatronics, 22(6), pp. 723-745.

7. Mohamed, M.K., Lanzon, A., 2012, Design and control of novel tri-rotor UAV, IEEE International Conference on Control (CONTROL) UKACC, pp. 304-309.

8. Ryll, M., Bülthoff, H.H., Giordano, P.R., 2015, A novel overactuated quadrotor unmanned aerial vehicle: Modeling, control, and experimental validation, IEEE Transactions on Control Systems Technology, 23(2), pp. $540-556$

9. Marconi, L., Naldi, R., Gentili, L., 2011, Modelling and control of a flying robot interacting with the environment, Automatica, 47(12), pp. 2571-2583.

10. Fernandes, N., 2011. Design and construction of a multi-rotor with various degrees of freedom, M.Sc. Thesis, Technical Univ. of Lisboa.

11. Elfeky, M., Elshafei, M., Saif, A.W.A., Al-Malki, M.F., 2013, Quadrotor helicopter with tilting rotors: Modeling and simulation, IEEE World Congress on Computer and Information Technology (WCCIT), pp. 1-5.

12. Elfeky, M., Elshafei, M., Saif, A.W.A., Al-Malki, M.F., 2016, Modeling and simulation of quadrotor UAV with tilting rotors, International Journal of Control, Automation and Systems, 14(4), pp. 1047-1055.

13. Badr, S., Mehrez, O., Kabeel, A.E., 2016, A novel modification for a quadrotor design, IEEE International Conference on Unmanned Aircraft Systems (ICUAS), pp. 702-710.

14. Alkamachi, A., Erçelebi, E., 2017, Modelling and Genetic Algorithm Based-PID Control of H-Shaped Racing Quadcopter, Arabian Journal for Science and Engineering, pp. 1-10, DOI: 10.1007/s13369-017-2433-2.

15. Hua, M. D., Hamel, T., Morin, P., Samson, C., 2013, Introduction to feedback control of underactuated VTOLvehicles: A review of basic control design ideas and principles, IEEE Control Systems, 33(1), pp. 61-75.

16. Gruber, D., 2000, The mathematics of the 3D rotation matrix, Xtreme Game Developers Conference.

17. Bayrakceken, M.K., Yalcin, M.K., Arisoy, A., Karamancioglu, A., 2011, HIL simulation setup for attitude control of a quadrotor, IEEE International Conference on Mechatronics (ICM), pp. 354-357. 
18. Fernando, H.C.T.E., De Silva, A.T.A., De Zoysa, M.D.C., Dilshan, K.A.D.C., Munasinghe, S.R., 2013, Modelling, simulation and implementation of a quadrotor UAV, 8th IEEE International Conference on Industrial and Information Systems (ICIIS), pp. 207-212.

19. Mahony, R., Kumar, V., Corke, P., 2012, Multirotor aerial vehicles, IEEE Robotics and Automation magazine, 19(3), pp. 20-32.

20. ElKholy, H.M., 2014, Dynamic modeling and control of a quadrotor using linear and nonlinear approaches, $\mathrm{PhD}$ dissertation, Master thesis, The American University in Cairo.

21. Mitchell, M., 1996, An introduction to genetic algorithms, MIT press.

22. von Frankenberg, F., 2016, Development of an autonomous omnicopter aerial vehicle, Master dissertation, Institute of Technology/ University of Ontario.

23. Beck, H., Lesueur, J., Charland-Arcand, G., Akhrif, O., Gagné, S., Gagnon, F., Couillard, D., 2016, Autonomous takeoff and landing of a quadcopter, IEEE International Conference on Unmanned Aircraft Systems (ICUAS), pp. 475-484. 